\title{
Sialolithotomy and sialodochoplasty of giant sialolith in the submandibular duct: a case report
}

\author{
Barra P. Novendra, Rahardjo, Poerwati S. Rahajoe*
}

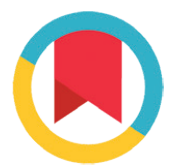

CrossMark

\section{Abstract}

Objective: To report a case of giant sialolith in the submandibular duct in which sialolithotomy and sialodochoplasty procedures were performed with an intraoral and extraoral approach.

Method: A 47 years old male patient reported a complaint of swelling in the left lower jaw which triggered discomfort, particularly when eating. The extraoral examination found $50 \mathrm{~mm}$ sized swelling that was firm in consistency, not painful and showed the same colour as the tissue in the left submandibular region. Meanwhile, the intraoral examination indicated a $5 \mathrm{~mm}$ sized whitish firm mass on the mucosal floor of the mouth. The panoramic radiograph examination showed a multiple radiopaque mass in the left mandible with a well-defined border. A multiple mass was found by the CT-scan examination in the left submandibular region and floor of the mouth. Sialographic examination described a total obstruction in the left submandibular duct and allegedly chronic inflammation-induced sialolith.

Results: Sialolithotomy was done with an intraoral and extraoral approach after which sialodochoplasty was also performed to insert the pediatric feeding tube no. 8 aiming to retain the shape and function of the submandibular duct.

Conclusion: Treating giant sialolith with sialolithotomy followed by sialodochoplasty has given a satisfactory result without any complication and recurrence.
Department of Oral and Maxillofacial Surgery, Faculty of Dentistry, Gadjah Mada University, Yogyakarta, Indonesia

*Corresponding to: Barra P. Novendra, Department of Oral and Maxillofacial Surgery, Faculty of Dentistry, Gadjah Mada University, Yogyakarta, Indonesia poerwati_soetji_fkg@ugm.ac.id

Received: 4 February 2018 Revised: 11 February 2018 Accepted: 19 February 2018 Available online 1 August 2018

Keywords: Sialolith, Submandibular Duct, Sialolitotomy, Sialodochoplasty

Cite this Article: Novendra BP, Rahardjo, Rahajoe PS. 2018. Sialolithotomy and sialodochoplasty of giant sialolith in the submandibular duct: a case report. Journal of Dentomaxillofacial Science 3(1): 119-122. D01: 10.15562/jdmfs.v3i2.712

\section{Introduction}

Sialolithiasis is a disorder of the salivary glands that allegedly occurs due to the accumulation of calcified calcium salt typed degenerative materials. The stone formed in the ductal system in the glands is called sialolith. ${ }^{1}$ Clinically, a sialolith is a round, oval, either rough or smooth, and yellowish. Sialolith content consists mostly of calcium phosphate, a little hydroxyapatite carbonate, magnesium, potassium and ammonia. ${ }^{2}$

Sialolith is generally $5-10 \mathrm{~mm}$ in size, above which is referred to as an unusual and rare sialolith. Giant sialolith refers to a sialolith exceeding $35 \mathrm{~mm}$ in size. ${ }^{3}$ The site and size of the sialolith determines the treatment to performed. Considerably varied, sialolith treatments can include non-surgical and surgical treatments. Simple treatments include a sialogogue treatment and massage to stimulate the stone to be pushed out. However, this method is rarely used given the high risk of recurrence and infection. Surgical treatments include sialolithotomy, sialodochotomy, sialodochoplasty and sialoadenectomy. ${ }^{1}$ Other possible non-surgical treatments include Extracorporeal Shock Wave Lithotripsy (ESWL) which is a shock wave-assisted non-invasive approach in a specific condition ${ }^{4}$

This paper reports a case of a $35 \mathrm{~mm}$ sized rare giant sialolith treated with sialolithotomy and sialodochoplasty with an extraoral and intraoral approach, where sialodochoplasty was performed by inserting a pediatric feeding tube no. 8 .

\section{Case Report}

A 47 years old male patient came to the oral surgery polyclinic of General Hospital Dr Sardjito, Yogyakarta with complaints of swelling in the lower left jaw. He was previously a week-hospitalized in the same hospital where he was diagnosed with a submandibular abscess due to sialolithiasis. He was treated with incision and drainage. The patient had lived with discomfort on the left floor of his mouth for the past 27 years, especially during meals. When examined by a surgeon, he was diagnosed with a salivary gland stone and recommended for a stone-removal operation to which he objected. A history of drug allergies, food allergies and systemic diseases was denied.

An extraoral examination showed swelling of the left submandible which was a $5 \mathrm{~cm}$ in diameter firm mass that was mobile, caused no pain when pressed, and was similar in colour with the surrounding tissues figure $1 \mathrm{~A}$ and $1 \mathrm{~B}$. Intraoral examination found a lesion at the left side of the mouth floor, adjacent to the caruncle, that was $5 \mathrm{~mm}$ in size, hard when palpated, showed no tenderness nor pus and the colour matched the surrounding tissue figure $1 \mathrm{C}$. 
Table 1 Cases of giant sialolith (>35 mm) from year 1942-2017

\begin{tabular}{|c|c|c|c|c|c|c|}
\hline No & Researchers & Number of cases & Age & Glands & Site & Size \\
\hline 1 & Meyers, 1942 & 1 & 50 & SM & Duct & 50 \\
\hline 2 & Mustard, 1945 & 1 & 42 & SM & Duct & 56 \\
\hline 3 & Allen, 1956 & 1 & 49 & SM & Duct & 35 \\
\hline 4 & Cavina and Santoli, 1965 & 1 & 59 & SM & Duct & 70 \\
\hline 5 & Cavina and Santoli & 1 & 53 & SM & Both & 60 \\
\hline 6 & Hoggins, 1968 & 1 & 52 & SM & Paren & 50 \\
\hline 7 & Rust and Messerly, 1969 & 1 & 66 & $\mathrm{P}$ & Duct & 51 \\
\hline 8 & Rust and Messerly, 1969 & 1 & 58 & NR & Paren & 35 \\
\hline 9 & Raksin et al., 1975 & 1 & 52 & SM & Duct & 55 \\
\hline 10 & Isacsson and Persson, 1982 & 1 & 48 & SM & Duct & 36 \\
\hline 11 & Tinsely, 1989 & 1 & 48 & SM & Paren & 50 \\
\hline 12 & Hubar et al., 1990 & 1 & 65 & SM & Duct & 52 \\
\hline 13 & Akin and Esmer, 1991 & 1 & 45 & SM & Paren & 45 \\
\hline 14 & Paul and Chauhan, 1995 & 1 & 45 & SM & Duct & 46 \\
\hline 15 & Bodner, 2002 & 1 & 50 & SM & Duct & 50 \\
\hline 16 & Ledesma-Montes et al., 2007 & 1 & 34 & SM & Duct & 36 \\
\hline 17 & Rai and Burman, 2009 & 1 & 60 & SM & Duct & 72 \\
\hline 18 & Huang et al., 2009 & 1 & 57 & SM & Duct & 40 \\
\hline 19 & Lokesh, 2011 & 1 & 50 & SM & Duct & 52 \\
\hline 20 & Manchil, 2013 & 1 & 47 & SM & Duct & 38 \\
\hline 21 & Fefar, 2015 & 1 & 46 & SM & Paren & 38 \\
\hline 22 & The current case, 2017 & 1 & 47 & SM & Duct & 51 \\
\hline
\end{tabular}

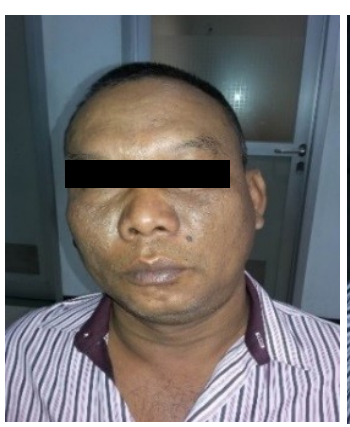

A

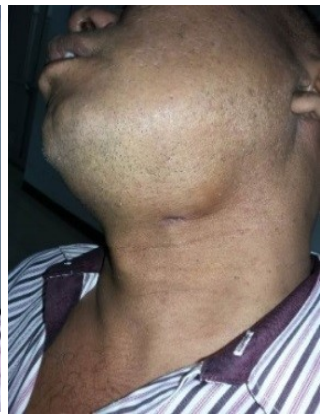

B

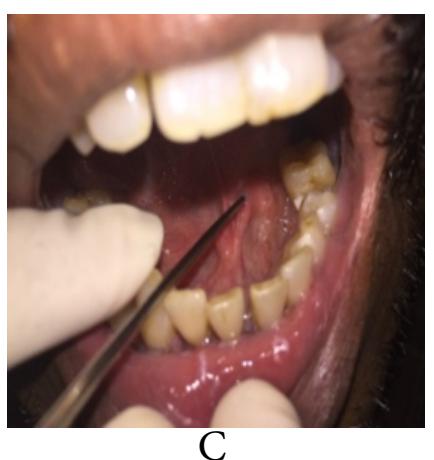

C
Figure 1 A. Extraoral facial condition, B. Swelling in the left submandible, C. Intraoral condition

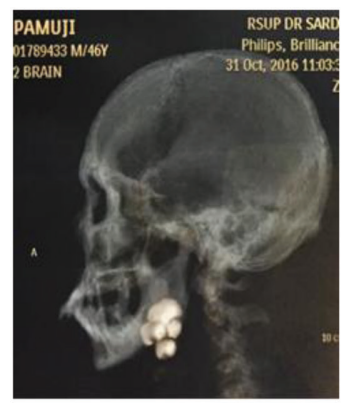

A

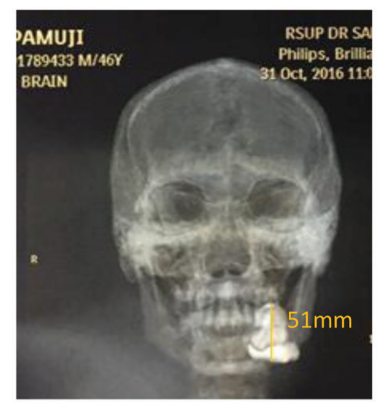

B

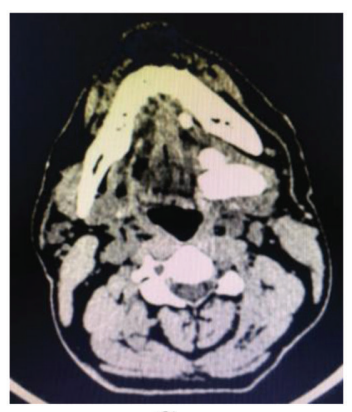

C
Figure 2 A. lateral CT scan, B. frontal CT scan, C. axial CT scan
An orthopantomogram (OPG) radiographic examination found an image of multiple masses in the left mandible that showed more radiopaque compared to the surroundings, varied in the shape and size with a well-defined border. Sialographic examination revealed the result of a total obstruction in the left submandibular duct was due to the suspected sialolith with chronic inflammation. A CT scan examination showed multiple masses on the mandible (4 overlapped masses in the left submandibular region and a mass in anterior close to the mouth floor) with a well-defined border and mandible bone separated, allegedly a sialolith figure 2A-2C.

The case was diagnosed as a left submandibular sialolithiasis. Sialolithotomy and sialodochoplasty under general anaesthesia were chosen for the treatment figure 3A-3I.

The patient stated no complaints during the control done one day after the surgery. An objective examination found no tongue or cheek paresthesia nor leakage of fluid coming out extraorally. One week following the surgery, the patient found that there was clear fluid that came out of the intraoral tube. Clinical examination on that day did not show any postoperative complications. When 


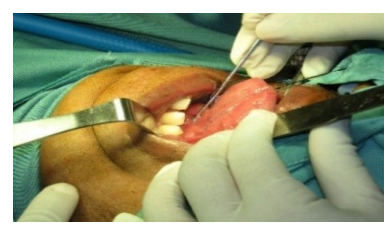

A

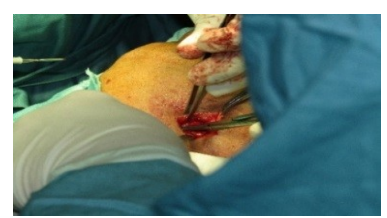

D

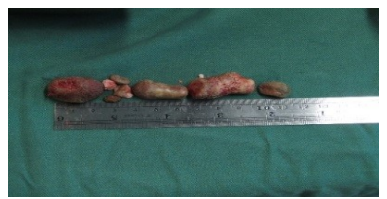

G

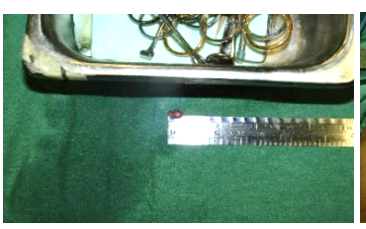

B

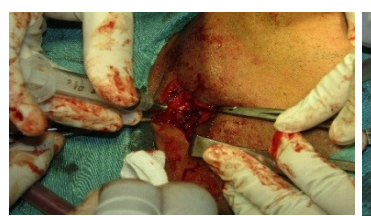

E

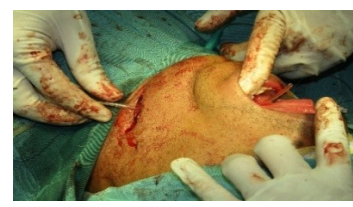

$\mathrm{H}$

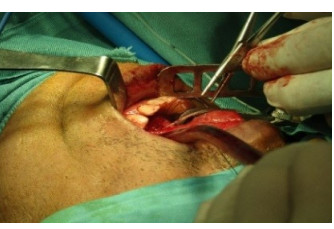

C

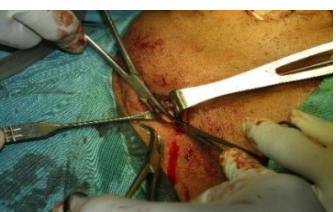

F

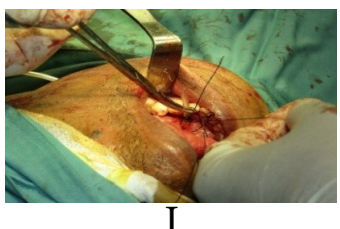

I
Figure 3 A. Identification of caruncle, B. Intraoral removal of sialolith, C. Intraoral sialodochoplasty, D. Extraoral sialolithotomy, E. Syringe is used as a guide, F. Extraorally-identified sialolith, G. Complete removal of sialolith, H. Extraoral sialodochoplasty with pediatric feeding tube no. 8 , I. Intraoral tube fixation

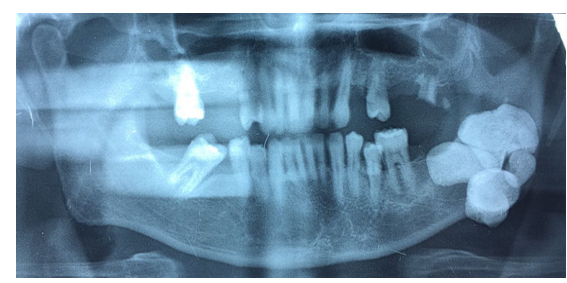

A

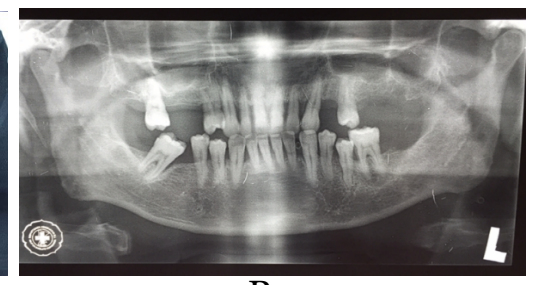

B
Figure 4 Panoramic radiography A. Preoperative, B. Postoperative

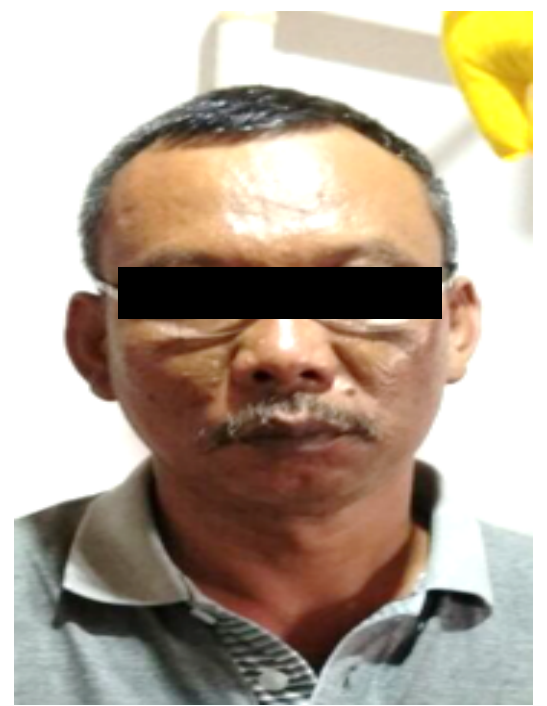

A

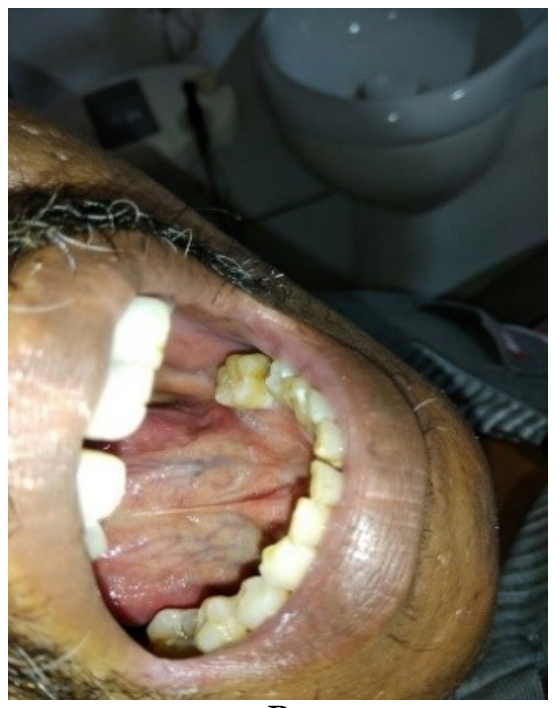

B
Figure 5 Eight months postoperative control: A. Extraoral, B. Intraoral with the production of saliva doing a postoperative control 2 weeks after the surgery, the patient felt a more comfortable mouth and the swelling disappeared. The sutures and tube were removed and the patient was instructed to consume acidic food to stimulate the saliva and keep the mouth clean. No residual sialolith was found based on the postoperative panoramic radiograph figure $4 \mathrm{~A}$ and $4 \mathrm{~B}$. Eight months postoperatively, the patient stated no pain complaints previously felt when eating, swelling disappeared, and the mouth was not dry figure $5 \mathrm{~A}$ and $5 \mathrm{~B}$.

The reported case was a $51 \mathrm{~mm}$-sized giant sialolith in its largest diameter. Sialolith, in this case, was plural and overlapped each other. The patient lived with the sialolith for 27 years and it stimulated the mass to develop. Journal articles of all the previously published giant sialolith cases over 35 $\mathrm{mm}$ in size were compiled by Manchil et al. ${ }^{5}$ and some other cases were also attached table 1.

\section{Discussion}

Giant sialolith is a rare case as sialolith as it is commonly a symptomatic case and therefore it receives treatment early. Sialolith may develop and grow to be a giant sialolith depending on how the salivary duct reacts. If the ducts are able to dilate and maintain the flow of saliva despite the growth of sialolith, an asymptomatic state will form for a long time, stimulating a giant sialolith to develop. ${ }^{6}$

The treatments of sialolithiasis are varied. The site and size of sialolith affects the choice of treatment. Large-sized and palpable sialolith can be removed using surgical procedures such as sialolithotomy and sialodochoplasty. ${ }^{5}$ Sialolith can be removed using a surgical procedure with an extraoral and intraoral approach. If the sialolith lies in the ducts and close to the papilla, an intraoral surgery can be carried out with longitudinal incisions of the ducts. An extraoral approach-based sialoadenectomy can be exercised if the sialolith lies inside the gland or deep into the mouth floor. ${ }^{7}$ The algorithm of submandibular sialolithiasis treatment depends on the site and size of sialolith. An intraoral sialolithotomy and sialodochoplasty is recommended for a sialolith in the submandibular ducts. ${ }^{1}$

Giant sialolith, in this case, is quite unique because it lies in the submandibular duct but is close to the submandibular glands and is more reachable by an extraoral than intraoral palpation. Besides, a smaller sialolith grows superficially adjacent to the caruncle. Referring to algorithm and different theories of sialolith, sialolithotomy should be the chosen treatment without sialoadenectomy, 
followed by an intraoral and extraoral approachbased sialodochoplasty.

Sialodochoplasty can be performed with or without a stent. The complication of sialodochoplasty without a stent includes a clogged duct by sutures with no guides, an occurrence of stenosis, and a delayed healing of the ductal wall due to a constant flow of saliva. Stenosis is a narrowing duct due to both physical and thermal trauma. A stent-assisted sialodochoplasty prevents postoperative stenosis and delayed duct healing. ${ }^{8}$ The types and sizes of stents vary which includes a pediatric feeding tube or a hypospadias silastic tube which is used in sialodochoplasty and sialendoscopy procedures. Su's et al. ${ }^{9}$ recommended a 2-week use of a pediatric feeding tube rather than a hypospadias silastic tube to prevent stenosis and infection.

Sialolithiasis, in this case, was treated with a pediatric feeding tube no. 8-assisted sialodochoplasty for two weeks, which resulted in no stenosis or infection marked with the saliva produced in the caruncle.

\section{Conclusion}

Giant sialolith is a rare case. The sialolithotomy and sialodochoplasty performed for this case have given satisfactory results without complications and recurrences. The site and size of sialolith determine the choice of treatment. Sialodochoplasty with a pediatric feeding tube can prevent the submandibular duct stenosis to occur.

\section{Acknowledgment}

The author would like to thank the patient who has been willing to share his case for reported and his cooperation to come for control treatment.

\section{Conflict of Interest}

The authors report no conflict of interest.

\section{References}

1. Carlson ER, Ord RA. Textbook and color atlas of salivary gland pathology-diagnosis and management. Sialolithiasis. Wiley- Blackwell; 2008. p. 109-122.

2. Williams MF. Sialolithiasis. Otolaryngol Clin North Am 1999;32: 819.

3. Rai M, Burman R. Giant submandibular sialolith of remarkable size in the comma area of Wharton's duct: a case report. J Oral Maxillofac Surg 2009;67: 1329-1332.

4. Pasquale C, Francesco O, Raffaele M, et al. Extracorporeal lithotripsy for salivary calculi: a long term clinical experience. Laryngoscope 2004;114: 1069-1073

5. Manchil RD, Kannan K. A giant sialolith in wharton's duct. J Indian Acad Oral Med Radiol 2013;25: 326-329.

6. Paul D, Chauhan MS. Salivary megalith with a sialocutanousand sialo-oral fistula: a case report. Eur Arch Otorhinolaryngol 1995; 109: 767.

7. Fefar AD, Jivani PS, Mehta MR. A huge salivary calculi of the submandibular gland: a case report with the review of literature. J Med Res 2015;1: 5-7.

8. Han PS, Kim Y, Yoo TS, et al. Sialodochoplasty stents: cost analysis and outcomes. J Oral \& Maxillofac Surg 2017;75: 536-542.

9. Su CH, Lee KS, Tseng TM. Post-sialendoscopy ductoplasty by salivary duct stent placements. Eur Arch Otorhinolaryngol 2016;273: 189-195.

10. Nurwahida, Hardianto A, Rizki KA. Multiple sialolithiasis of submandibular gland: a case report. J Dentomaxillofac Sci 2017;2: 129-132.

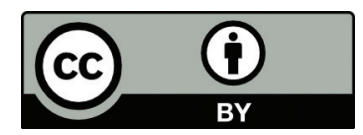

This work is licensed under a Creative Commons Attribution 